04

\title{
Четырехволновое взаимодействие на фазово-амплитудных голографических решетках в фоторефрактивном пьезокристалле класса симметрии $\overline{\mathbf{4}} 3 \mathrm{~m}$
}

\author{
(C) В.Н. Навныко \\ Мозырский государственный педагогической университет имени И.П. Шамякина, \\ 247760 Мозырь, Гомельская обл., Республика Беларусь \\ e-mail: valnav@inbox.ru
}

Поступила в редакцию 16.11.2021 г.

В окончательной редакции 16.11.2021 г.

Принята к публикации 18.12.2021 г.

\begin{abstract}
Представлена система уравнений связанных волн, пригодная для расчета векторных амплитуд линейно поляризованных световых волн при четырехволновом взаимодействии на фазово-амплитудных голографических решетках в кубическом фоторефрактивном полупроводнике произвольного среза, принадлежащем классу симметрии $\overline{4} 3 m$. На основании численного решения системы уравнений связанных волн рассчитаны графики зависимости интенсивностей поляризационных компонент обращенной световой волны от ориентационного угла для кристалла GaAs среза (110). Проведено сравнение полученных графиков зависимостей с известными теоретическими и экспериментальными данными. Показано, что наилучшее совпадение результатов теоретического моделирования и экспериментальных данных достигается в случае, если при расчете встречного четырехволнового взаимодействия в кристалле GaAs среза (110) допускается формирование нескольких фазово-амплитудных голографических решеток, а также принимается во внимание вклад фотоупругого и обратного пьезоэлектрического эффектов вместе с поглощением кристалла.
\end{abstract}

Ключевые слова: четырехволновое взаимодействие, фоторефрактивный полупроводник, световая волна, голографическая решетка, уравнения связанных волн.

DOI: $10.21883 /$ OS.2022.03.52167.2936-21

\section{Введение}

Фоторефрактивные кристаллы могут использоваться в качестве светочувствительных материалов в устройствах, предназначенных для генерации, обработки и передачи оптических сигналов [1]. Вследствие наличия стационарного усиления падающего светового пучка и возможности создания обратной связи, обеспечивающей обращение волнового фронта, фоторефрактивные кристаллы используются при разработке лазеров на динамических решетках [2]. Кубические фоторефрактивные полупроводники обладают рядом преимуществ по отношению к другим фоточувствительным материалам, поскольку обладают относительно малым временем фоторефрактивного отклика, низким порогом генерации по мощности волн накачки и возможностью перехода в инфракрасный диапазон спектра [3].

Результаты пионерских исследований обращения волнового фронта при четырехволновом взаимодействии в фоторефрактивных полупроводниках доложены в работах [4-7]. Результаты экспериментального наблюдения вырожденного четырехволнового взаимодействия в кристалле GaAs:EL2 приведены в [4]. В работе экспериментально определено время фоторефрактивного отклика и предсказана возможность использования кристаллов GaAs в оптических устройствах, от которых требуется высокая скорость и чувствительность. Практически одновременно выходит работа [5], в которой приведены результаты экспериментального изучения закономерностей четырехволнового взаимодействия в кристалле $\mathrm{InP}: \mathrm{Fe}$. B рамках этой работы исследовано влияние приложенного к кристаллу переменного электрического поля на интенсивность обращенной световой волны и указано на возможность использования таких кристаллов для обработки оптических сигналов в инфракрасном диапазоне. В [6] изучено влияние приложенного переменного электрического поля на коэффициент отражения при четырехволновом взаимодействии в кристалле $\mathrm{GaAs}$, а также изучены характеристики обращенного волнового фронта. Наибольший экспериментально измеренный стационарный коэффициент отражения для кристалла GaAs составил 15\%, причем наблюдалось кратковременное увеличение коэффициента отражения до 510\%. В [7] продемонстрировано, что при записи бегущих голографических решеток и при приложении к кристаллу GaAs: Cr постоянного внешнего электрического поля коэффициент отражения может достигать 500\%.

Наряду с исследованиями закономерностей обращения волнового фронта в фоторефрактивных полупроводниках изучаются $[8,9]$ возможности использования таких кристаллов в устройствах, предназначенных для обработки изображений и в оптических генераторах. В [8] представлены результаты экспериментов по об- 
ращению волнового фронта и обработке изображений. Показано, что кристаллы GaAs, несмотря на относительно малый электрооптический коэффициент, могут эффективно использоваться как регистрирующие среды в устройствах, предназначенных для обработки изображений. Закономерности четырехволнового взаимодействия в $\mathrm{Ga} / \mathrm{AlGaAs}$ полупроводниковых лазерах проанализированы в [9]. Полученные результаты демонстрируют, что кристаллы $\mathrm{Ga} / \mathrm{AlGaAs}$ могут являться эффективным источником нелинейного оптического взаимодействия в лазерах. В настоящее время четырехволновое взаимодействие применяется для изучения и совершенствования полупроводниковых лазеров на квантовых точках (см., например, [10-12]).

Теоретический анализ дифракции световых волн на голографических решетках в кубических фоторефрактивных кристаллах достаточно сложен, поскольку помимо линейного электрооптического эффекта требует дополнительного учета ряда свойств регистрирующей среды. При записи и восстановлении голографической решетки в фоторефрактивном пьезокристалле существенную роль играют упругие деформации, возникающие при наведении электрических полей [13]. Наличие упругих деформаций обусловливает необходимость дополнительного учета фотоупругого и обратного пьезоэлектрического эффектов при расчете модуляции диэлектрической проницаемостью кристалла на оптических частотах. Расчет анизотропии голографической решетки в кубическом кристалле с одновременным учетом линейного электрооптического, фотоупругого и обратного пьезоэлектрического эффектов выполнен в [14]. При расчете эффективности дифракции на объемной голограмме также следует учитывать, что голографическая решетка может иметь фазово-амплитудную структуру (смешанная голограмма) и представлять собой совокупность фазовой и амплитудной решеток $[15,16]$. Фазовая решетка возникает за счет пространственной модуляции показателя преломления регистрирующей среды, а амплитудная решетка - показателя поглощения. Вклад амплитудной составляющей может оказывать существенное влияние на дифракционную эффективность голографической решетки, сформированной в кубическом фоторефрактивном кристалле [17]. В работе показано, что наилучшее согласование теоретических и экспериментальных данных, полученных при нахождении ориентационной зависимости дифракционной эффективности пропускающей голограммы, сформированной в кристалле $\mathrm{Bi}_{12} \mathrm{TO}_{20}$ (ВТО), достигается при учете фазово-амплитудной структуры голографической решетки. В [18] описана экспериментальная методика раздельного изучения свойств фазовых и амплитудных голографических решеток в кристаллах GaAs и InP, которая основывается на изучении динамики изменения интенсивности дифрагированного пучка при четырехволновом взаимодействии. Показано, что начальное быстрое уменьшение интенсивности дифрагированного светового пучка обусловлено стиранием амплитудной голографической решетки, а последующее плавное уменьшение интенсивности вызвано стиранием фазовой голографической решетки.

В ранних теоретических работах при расчете четырехволнового взаимодействия в кубических фоторефрактивных кристаллах пренебрегалось совместным вкладом фотоупругого и обратного пьезоэлектрического эффектов, фазово-амплитудной структурой голограмм и поглощением, что могло приводить к заметным отклонениям теоретических данных от результатов эксперимента [19]. Расчет оптимальных условий обращения волнового фронта в кубических фоторефрактивных кристаллах класса симметрии 23 с учетом фотоупругого и обратного пьезоэлектрического эффектов, оптической активности и поглощения выполнен в [20]. На основании решения уравнений связанных волн в работе найдены аналитические выражения для коэффициентов прохождения и отражения при четырехволновом взаимодействии на пропускающей голографической решетке с волновым вектором, ориентированном вдоль кристаллографического направления [110], и световыми волнами, волновые нормали которых лежат в плоскости (001). Рассчитаны пороговые условия генерации обращенного светового фронта при взаимодействии неортогонально поляризованных световых пучков накачки при приложении к кристаллу внешнего меандрового электрического поля. Анализ закономерностей векторного четырехволнового взаимодействия на отражательной голографической решетке в кубическом оптически активном фоторефрактивном пьезокристалле выполнен в [21]. В работе теоретически предсказано, что для кристалла ВТО:Fe, $\mathrm{Cu}$ толщиной более $3 \mathrm{~mm}$ коэффициент отражения может превышать 100\% при оптимальном выборе ориентаций вектором поляризаций сигнальной и накачивающих волн. Представлена система уравнений связанных волн, пригодная для расчета обращенного волнового фронта при дифракции световых пучков на отражательной голографической решетке в кубическом фоторефрактивном кристалле класса симметрии 23 произвольного среза. Особенности четырехволнового взаимодействия на фазово-амплитудных пропускающих решетках в кристалле ВТО среза (110) с учетом фотоупругого и обратного пьезоэлектрического эффектов, оптической активности, поглощения и циркулярного дихроизма изучены в [22]. Теоретически изучена зависимость интенсивности обращенной световой волны от ориентационного угла и толщины кристалла. Экспериментально установлено, что в кристалле ВТО толщиной $7.7 \mathrm{~mm}$ коэффициент отражения может достигать 240\%. В работе определены значения ориентационных углов, соответствующих наибольшим значениям коэффициента отражения. Показано, что наилучшее согласование теоретических и экспериментальных данных достигается в случае, если при расчетах принимается во внимание фазовоамплитудная структура формируемых в кристалле ВТО пропускающих голографических решеток.

Задача об анализе закономерностей обращения волнового фронта на фазово-амплитудных голографических 
решетках в кубических фоторефрактивных кристаллах класса симметрии $\overline{4} 3 m$ с учетом фотоупругого и обратного пьезоэлектрического эффектов, насколько нам известно, до настоящего времени не рассматривалась. При решении этой задачи следует учитывать, что в общем случае при встречном четырехволновом взаимодействии в кубическом фоторефрактивном кристалле может одновременно формироваться 6 фазово-амплитудных голографических решеток [2]. Учет в теоретической модели указанных особенностей позволит найти новые закономерности формирования обращенного волнового фронта при четырехволновом взаимодействии в фоторефрактивном полупроводнике, более точно рассчитать коэффициенты отражения и прохождения, а также предсказать оптимальные условия генерации сопряженной по фазе световой волны.

Целью настоящего исследования было построение математической модели, пригодной для описания вырожденного встречного четырехволнового взаимодействия на фазово-амплитудных голографических решетках, формируемых в кубическом фоторефрактивном кристалле класса симметрии $\overline{4} 3 m$, и сравнение получаемых на основании этой модели теоретических данных с результатами известных экспериментальных исследований. При разработке математической модели рассмотрен случай, когда при четырехволновом взаимодействии в фоторефрактивном кристалле формируется 6 фазово-амплитудных голографических решеток, а взаимодействующие волны имеют линейную поляризацию. В теоретической модели также будут приняты во внимание линейный электрооптический, фотоупругий и обратный пьезоэлектрический эффекты, а также поглощение кристалла.

\section{Математическая модель}

Пусть на кубический фоторефрактивный полупроводник падают две волны накачки 1 и 2 , а также сигнальная волна 3 (рис. 1). Предположим, что световые волны являются монохроматическими линейно поляризованными и распространяются в плоскости падения (I). Волны накачки 1 и 2 распространяются в направлениях, обозначенных единичными векторами $\mathbf{e}_{1 n}$ и $\mathbf{e}_{2 n}$. Направление распространения сигнальной волны совпадает по направлению с единичным вектором $\mathbf{e}_{3 n}$. Волна 4 возникает в результате дифракционных процессов волн накачки и сигнальной волны на сформированных в кристалле голографических решетках и распространяется в направлении единичного вектора $\mathbf{e}_{4 n}$.

Для описания состояния поляризации $j$-й волны используем ортонормированный базис $\left(\mathbf{e}_{j p}, \mathbf{e}_{j s}, \mathbf{e}_{j n}\right)$, где $\mathbf{e}_{j n}=\left[\mathbf{e}_{j p} \times \mathbf{e}_{j s}\right](j=1,2,3,4)$. Вектор $\mathbf{e}_{j p}$ лежит в плоскости падения и используется для задания $p$-поляризации $j$-й волны. Вектор $\mathbf{e}_{j s}$ перпендикулярен плоскости падения и используется для задания $s$-поляризации $j$-й волны. Вектор $\mathbf{e}_{j n}$ совпадает по направлению с волновой нормалью $j$-й волны. Вектор $\mathbf{E}_{j}$ является вектором напряженности электрического поля $j$-й волны и лежит в плоскости, содержащей векторы $\mathbf{e}_{j p}$ и $\mathbf{e}_{j s}$. Для задания ориентации вектора $\mathbf{E}_{j}$ относительно кристалла используется азимут поляризации $\psi_{j}$, который является мерой углового расстояния между векторами $\mathbf{e}_{j p}$ и $\mathbf{E}_{j}$, отсчитываемый по часовой стрелке, если смотреть вдоль вектора $\mathbf{e}_{j n}$.

Поверхность голографического стола параллельна плоскости (I), с которой жестко связан ортонормированный базис $\left(\mathbf{e}_{1}, \mathbf{e}_{2}, \mathbf{e}_{3}\right)$, где $\mathbf{e}_{3}=\left[\mathbf{e}_{1} \times \mathbf{e}_{2}\right]$. На рис. 1 векторы $\mathbf{e}_{1}$ и $\mathbf{e}_{3}$ лежат в плоскости (I). Координата $z$ отсчитывается вдоль положительного направления оси $O z$, совпадающего по направлению с единичным вектором $\mathbf{e}_{3}$. За начало отсчета оси $O z \quad(z=0)$ принимается точка ее пересечения с гранью кристалла (II). Кристалл можно поворачивать вокруг оси $O z$, и его положение относительно голографического стола определяется с использованием единичных векторов $\mathbf{s}$ и $\mathbf{q}$, которые жестко фиксированы в кристаллографической системе координат. Ориентационный угол $\theta$ используется для задания угла поворота кристалла относительно оси $O z$ и определяется как угловое расстояние между векторами $\mathbf{e}_{2}$ и $\mathbf{q}$, которое отсчитывается по часовой стрелке в плоскости (II) от вектора $\mathbf{e}_{2}$ к вектору $\mathbf{q}$, если смотреть навстречу вектору е 3 . Будем считать, что грань (II) кристалла и параллельная ей грань являются рабочими и покрыты антиотражающим покрытием. Расстояние между этими гранями равняется толщине кристалла $d$, которая отсчитывается от грани (II). Угловое расстояние между вектором $\mathbf{e}_{j n}$ и осью $O z$ обозначается как угол $\varphi_{j}$, который отсчитывается в плоскости падения и равняется углу Брэгга $\varphi$.

На вставке рис. 1 в рабочей системе координат отображена ориентация волновых векторов двух пропускающих $\left(\mathbf{K}_{13}, \mathbf{K}_{24}\right)$ и четырех отражательных $\left(\mathbf{K}_{12}, \mathbf{K}_{14}, \mathbf{K}_{23}, \mathbf{K}_{34}\right)$ голографических решеток, которые могут формироваться при встречном четырехволновом взаимодействии в фоторефрактивном кристалле. Пропускающие голограммы 13 и 24 образуются в результате попарной интерференции соответственно волны накачки 1 с сигнальной волной 3 и волны накачки 2 с волной 4. Отражательные голографические решетки 14 и 23 формируются при попарном взаимодействии волны накачки 1 с волной 4 и волны накачки 2 с сигнальной волной 3. Интерференция волн накачки 1 и 2 обусловливает формирование отражательной голограммы $12.3 \mathrm{a}$ счет когерентного взаимодействия сигнальной волны 3 с волной 4 может происходить образование отражательной голограммы 34.

Стационарное решение волнового уравнения будем искать в виде суперпозиции четырех линейно поляризованных световых волн:

$$
\mathbf{E}=\sum_{j=1}^{4}\left(E_{j p} \mathbf{e}_{j p}+E_{j s} \mathbf{e}_{j s}\right) e^{i \vartheta_{j}},
$$




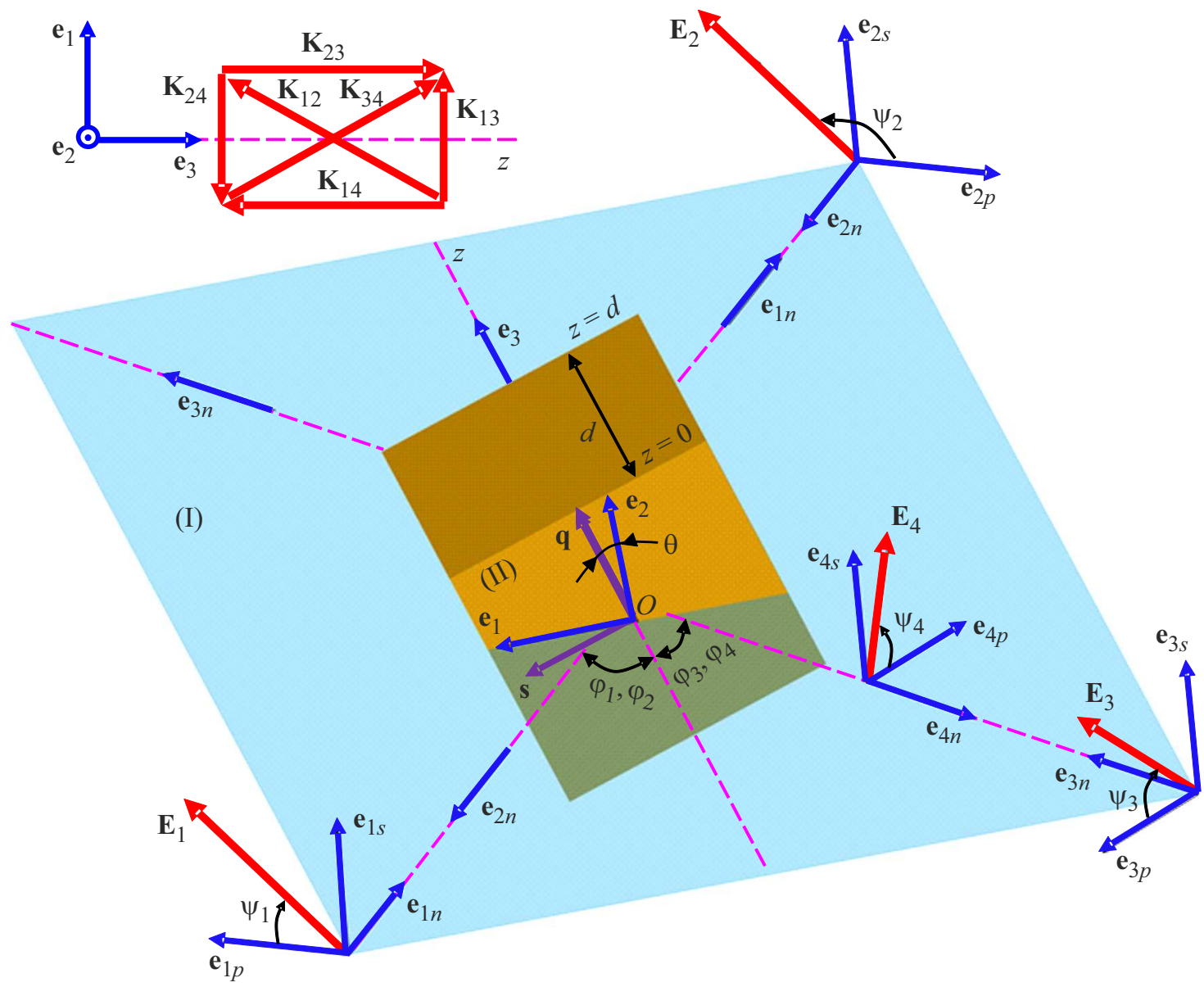

Рис. 1. Схема встречного четырехволнового взаимодействия в фоторефрактивном полупроводнике.

где $\mathbf{E}$ - вектор электрической напряженности результирующего светового поля в кристалле, изменяющийся вдоль оси $z ; E_{j p}, E_{j s}-p$ - и $s$-компоненты векторной амплитуды $\mathbf{E}_{j}$, являющиеся функциями координаты $z$; $\vartheta_{j}$ - начальная фаза $j$-й волны.

Когерентное взаимодействие световых волн приводит к образованию интерференционных картин в кристалле. Глубина модуляции интерференционных картин может быть найдена на основании следующих выражений:

$$
m^{h u}=\left(E_{h s} E_{u s}+E_{h p} E_{u p} \cos \left(\mathbf{e}_{h p} \mathbf{e}_{u p}\right)\right) / I_{0},
$$

где $m^{h u}$ - глубина модуляции интерференционной картины $h u(h u=12,13,14,23,24,34)$; $\left(\mathbf{e}_{h p} \mathbf{e}_{u p}\right)$ - скалярное произведение векторов. Здесь и далее верхний индекс $h u$ означает, что параметр находится применительно к голографической решетке $h u$. Результирующая интенсивность светового поля внутри кристалла $I_{0}$ находится по формуле $I_{0}=E_{1 s}^{2}+E_{1 p}^{2}+E_{2 s}^{2}+E_{2 p}^{2}+E_{3 s}^{2}+E_{3 p}^{2}+E_{4 s}^{2}+E_{4 p}^{2}$.

С использованием выражений (1), (2) из волнового уравнения в приближении медленно меняющихся амплитуд можно получить уравнения связанных волн, которые в дальнейшем используются для анализа закономерностей встречного четырехволнового взаимодействия на двух пропускающих и четырех отражательных фазовоамплитудных решетках, сформированных в фоторефрактивном кристалле класса симметрии $\overline{4} 3 m$ произвольного среза:

$$
\begin{aligned}
& \frac{d E_{1 p}}{d z}=\left(\kappa_{1 p 2 p}+\sigma_{1 p 2 p}\right) E_{2 p}+\left(\kappa_{1 p 2 s}+\sigma_{1 p 2 s}\right) E_{2 s} \\
& +\left(\kappa_{1 p 3 p}+\sigma_{1 p 3 p}\right) E_{3 p}+\left(\kappa_{1 p 3 s}+\sigma_{1 p 3 s}\right) E_{3 s} \\
& +\left(\kappa_{1 p 4 p}+\sigma_{1 p 4 p}\right) E_{4 p}+\left(\kappa_{1 p 4 s}+\sigma_{1 p 4 s}\right) E_{4 s}-\alpha_{1} E_{1 p},
\end{aligned}
$$

$$
\begin{aligned}
& \frac{d E_{1 s}}{d z}=\left(\kappa_{1 s 2 p}+\sigma_{1 s 2 p}\right) E_{2 p}+\left(\kappa_{1 s 2 s}+\sigma_{1 s 2 s}\right) E_{2 s} \\
& +\left(\kappa_{1 s 3 p}+\sigma_{1 s 3 p}\right) E_{3 p}+\left(\kappa_{1 s 3 s}+\sigma_{1 s 3 s}\right) E_{3 s} \\
& +\left(\kappa_{1 s 4 p}+\sigma_{1 s 4 p}\right) E_{4 p}+\left(\kappa_{1 s 4 s}+\sigma_{1 s 4 s}\right) E_{4 s}-\alpha_{1} E_{1 s},
\end{aligned}
$$

$$
\begin{aligned}
& \frac{d E_{2 p}}{d z}=\left(-\kappa_{2 p 1 p}+\sigma_{2 p 1 p}\right) E_{1 p}+\left(-\kappa_{2 p 1 s}+\sigma_{2 p 1 s}\right) E_{1 s} \\
& +\left(\kappa_{2 p 3 p}+\sigma_{2 p 3 p}\right) E_{3 p}+\left(\kappa_{2 p 3 s}+\sigma_{2 p 3 s}\right) E_{3 s} \\
& +\left(\kappa_{2 p 4 p}+\sigma_{2 p 4 p}\right) E_{4 p}+\left(\kappa_{2 p 4 s}+\sigma_{2 p 4 s}\right) E_{4 s}-\alpha_{2} E_{2 p},
\end{aligned}
$$




$$
\begin{aligned}
& \frac{d E_{2 s}}{d z}=\left(-\kappa_{2 s 1 p}+\sigma_{2 s 1 p}\right) E_{1 p}+\left(-\kappa_{2 s 1 s}+\sigma_{2 s 1 s}\right) E_{1 s} \\
& +\left(\kappa_{2 s 3 p}+\sigma_{2 s 3 p}\right) E_{3 p}+\left(\kappa_{2 s 3 s}+\sigma_{2 s 3 s}\right) E_{3 s} \\
& +\left(\kappa_{2 s 4 p}+\sigma_{2 s 4 p}\right) E_{4 p}+\left(\kappa_{2 s 4 s}+\sigma_{2 s 4 s}\right) E_{4 s}-\alpha_{2} E_{2 s}, \\
& \frac{d E_{3 p}}{d z}=\left(-\kappa_{3 p 1 p}+\sigma_{3 p 1 p}\right) E_{1 p}+\left(-\kappa_{3 p 1 s}+\sigma_{3 p 1 s}\right) E_{1 s} \\
& +\left(-\kappa_{3 p 2 p}+\sigma_{3 p 2 p}\right) E_{2 p}+\left(-\kappa_{3 p 2 s}+\sigma_{3 p 2 s}\right) E_{2 s} \\
& +\left(\kappa_{3 p 4 p}+\sigma_{3 p 4 p}\right) E_{4 p}+\left(\kappa_{3 p 4 s}+\sigma_{3 p 4 s}\right) E_{4 s}-\alpha_{3} E_{3 p} \\
& \frac{d E_{3 s}}{d z}=\left(-\kappa_{3 s 1 p}+\sigma_{3 s 1 p}\right) E_{1 p}+\left(-\kappa_{3 s 1 s}+\sigma_{3 s 1 s}\right) E_{1 s} \\
& +\left(-\kappa_{3 s 2 p}+\sigma_{3 s 2 p}\right) E_{2 p}+\left(-\kappa_{3 s 2 s}+\sigma_{3 s 2 s}\right) E_{2 s} \\
& +\left(\kappa_{3 s 4 p}+\sigma_{3 s 4 p}\right) E_{4 p}+\left(\kappa_{3 s 4 s}+\sigma_{3 s 4 s}\right) E_{4 s}-\alpha_{3} E_{1 s} \\
& \frac{d E_{4 p}}{d z}=\left(-\kappa_{4 p 1 p}+\sigma_{4 p 1 p}\right) E_{1 p}+\left(-\kappa_{4 p 1 s}+\sigma_{4 p 1 s}\right) E_{1 s} \\
& +\left(-\kappa_{4 p 2 p}+\sigma_{4 p 2 p}\right) E_{2 p}+\left(-\kappa_{4 p 2 s}+\sigma_{4 p 2 s}\right) E_{2 s} \\
& +\left(-\kappa_{4 p 3 p}+\sigma_{4 p 3 p}\right) E_{3 p}+\left(-\kappa_{4 p 3 s}+\sigma_{4 p 3 s}\right) E_{3 s}-\alpha_{4} E_{4 p} \\
& \frac{d E_{4 s}}{d z}=\left(-\kappa_{4 s 1 p}+\sigma_{4 s 1 p}\right) E_{1 p}+\left(-\kappa_{4 s 1 s}+\sigma_{4 s 1 s}\right) E_{1 s} \\
& \left.+\sigma_{4 s 2 p}\right) E_{2 p}+\left(-\kappa_{4 s 2 s}+\sigma_{4 s 2 s}\right) E_{2 s} \\
& \left.+\sigma_{4 s 2 p}\right) E_{3 p}+\left(-\kappa_{4 s 3 s}+\sigma_{4 s 3 s}\right) E_{3 s}-\alpha_{4} E_{4 s} \\
& +(9) \\
& +\kappa_{4}
\end{aligned}
$$

В уравнениях связанных волн приняты следующие обозначения: $\kappa_{h b u t}=\left(\kappa_{0}\left(\mathbf{e}_{h b} \Delta \hat{\eta}^{h u} \mathbf{e}_{u t}\right)\right) / \cos \varphi_{h}$, $\sigma_{h b u t}=\left(\sigma_{0}\left(\mathbf{e}_{h b} \Delta \hat{\sigma}^{h u} \mathbf{e}_{u t}\right)\right) / \cos \varphi_{h}, \quad \alpha_{h}=\alpha / \cos \varphi_{h}, \quad$ где $\kappa_{0}=\pi n_{0}^{3} /(2 \lambda)-$ постоянная связи фазовой голографической решетки, $\sigma_{0}=-\pi /(n c)-$ постоянная связи амплитудной голографической решетки, $\Delta \hat{\eta}^{h u}-$ возмущения обратного тензора диэлектрической проницаемости кристалла, соответствующие фазовой голографической решетке $h u, \Delta \hat{\sigma}^{h u}$ - возмущения тензора проводимости кристалла, соответствующие амплитудной голографической решетке $h u, n_{0}$ - показатель преломления невозмущенного кристалла, $\alpha-$ коэффициент естественного поглощения кристалла, $\lambda$ - длина волны, $c-$ скорость света в вакууме; $h, u=1,2,3,4 ; b, t=p, s$. Выражения для нахождения возмущений обратного тензора диэлектрической проницаемости $\Delta \hat{\eta}^{13,24}$ кубического кристалла с учетом совместного действия фотоупругого и обратного пьезоэлектрического эффектов приведены в [23]. Возмущения тензора проводимости кубического кристалла в линейном по контрасту приближении рассчитываются на основании следующего выражения: $\Delta \hat{\sigma}^{h u}=m^{h u} \sigma^{h u} \delta_{k n}$, где $\sigma^{h u}-$ коэффициенты проводимости, $\delta_{k n}-$ единичный симметричный тензор второго ранга. При получении уравнения связанных волн (3)-(10) предполагалось, что пространственные сдвиги фазовых и амплитудных решеток относительно соответствующих интерференционных картин равны $\pi / 2$ и 0 соответственно.
При решении уравнений связанных волн (3)-(10) использовались параметры кристалла $\mathrm{GaAs}$, соответствующие длине волны $\lambda=1064 \cdot 10^{-9} \mathrm{~m}$ и заимствованные из работ [19,24,25]: показатель поглощения $\alpha=410 \mathrm{~m}^{-1}$ [19]; показатель преломления невозмущенного кристалла $n_{0}=3.48$ [24]; электрооптический коэффициент $r_{41}=-1.43 \cdot 10^{-12} \mathrm{~m} / \mathrm{V}$ [24]; коэффициенты упругости $c_{1}=11.88 \cdot 10^{10} \mathrm{~N} / \mathrm{m}^{2}, c_{2}=5.38 \cdot 10^{10} \mathrm{~N} / \mathrm{m}^{2}$, $c_{3}=5.94 \cdot 10^{10} \mathrm{~N} / \mathrm{m}^{2}$ [24]; коэффициенты фотоупругости $p_{1}=-0.165, \quad p_{2}=p_{3}=-0.14, \quad p_{4}=-0.072 \quad[25]$; пьезоэлектрический коэффициент $e_{14}=0.154 \mathrm{C} / \mathrm{m}^{2}[24]$. Здесь приняты следующие обозначения для ненулевых компонент тензоров линейного электрооптического $\left(\hat{r}^{S}\right)$, фотоупругого $\left(\hat{p}^{E}\right)$ и обратного пьезоэлектрического $(\hat{e})$ эффектов, а также компонент тензора упругости $\left(\hat{c}^{E}\right)$ : $r_{123}^{S}=r_{132}^{S}=r_{213}^{S}=r_{231}^{S}=r_{312}^{S}=r_{321}^{S} \equiv r_{41}, p_{11}^{E}=p_{22}^{E}=$ $=p_{33}^{E} \equiv p_{1}, \quad p_{12}^{E}=p_{23}^{E}=p_{31}^{E} \equiv p_{2}, \quad p_{13}^{E}=p_{21}^{E}=p_{32}^{E} \equiv$ $\equiv p_{3}, \quad p_{44}^{E}=p_{55}^{E}=p_{66}^{E} \equiv p_{4}, \quad e_{123}=e_{132}=e_{123}=e_{231}=$ $=e_{312}=e_{321} \equiv e_{14}, \quad c_{11}^{E}=c_{22}^{E}=c_{33}^{E} \equiv c_{1}, \quad c_{12}^{E}=c_{13}^{E}=$ $=c_{23}^{E}=c_{21}^{E}=c_{31}^{E}=c_{32}^{E} \equiv c_{2}, \quad c_{44}^{E}=c_{55}^{E}=c_{66}^{E} \equiv c_{3}$. Индекс $S$ для тензора линейного электрооптического эффекта $\hat{r}^{S}$ означает, что компонента $r_{41}$ тензора линейного электрооптического эффекта измерялась для зажатого кристалла; компоненты тензоров упругости $\hat{c}^{E}$ и фотоупругого эффекта $\hat{p}^{E}$ измерялись при постоянном электрическом поле. При расчетах отношения интенсивностей световых волн и значение угла Брэгга $\varphi_{B}$ в воздухе выбирались на основании данных, представленных в [19]. Начальные условия для решения двухточечной граничной задачи выбирались следующим образом: $\quad E_{1 p}(0)=E_{1} \cos \psi_{1}, \quad E_{1 s}(0)=E_{1} \sin \psi_{1}$, $E_{2 p}(d)=E_{2} \cos \psi_{2}, E_{2 s}(d)=E_{2} \sin \psi_{2}, E_{3 p}(0)=E_{3} \cos \psi_{3}$, $E_{3 s}(0)=E_{3} \sin \psi_{3}, E_{4 p}(d)=0, E_{4 s}(d)=0$. Численное решение уравнений связанных волн $(3)-(10)$ выполнялось с использованием известного метода стрельбы [26].

\section{Полученные результаты и их анализ}

На рис. 2 и 3 представлены графики зависимостей интенсивностей $p$-поляризованной $\left(I_{4 p}\right.$ на рис. $2, a$ и рис. $3, b)$ и $s$-поляризованной $\left(I_{4 s}\right.$ на рис. $2, b$ и рис. $\left.3, a\right)$ компонент волны 4 от ориентационного угла $\theta$, которые получены теоретическим и экспериментальным способами при изучении встречного четырехволнового взаимодействия в кристалле GaAs среза (110) толщиной $d=1.5 \cdot 10^{-3} \mathrm{~m}$. Данные на рис. 2 и 3 получены для случая, когда волна накачки 1 и сигнальная волна 3 имеют одинаковую $s$-поляризацию, а волна накачки 2 поляризована либо в плоскости падения (рис. 2), либо перпендикулярно ей (рис. 3). Темные точки на рисунках соответствуют приведенным в [19] экспериментальным данным и отображают ориентационные зависимости $I_{4 p}(\theta)$ и $I_{4 s}(\theta)$. Штриховые линии на рис. 2 и 3 соответствуют графикам зависимостей $I_{4 p}(\theta)$ и $I_{4 s}(\theta)$, полученным в [19] на основании аналитического решения уравнений связанных волн. 

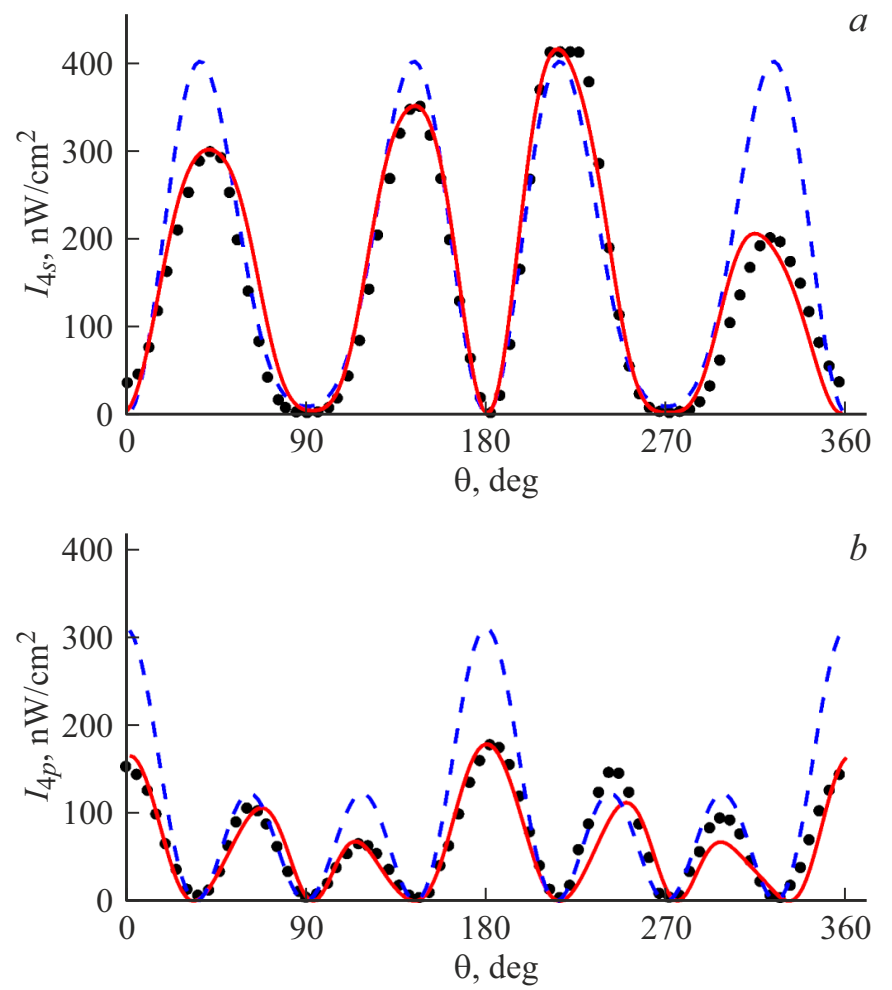

Рис. 2. Зависимости интенсивностей $p$-поляризованной $(a)$ и $s$-поляризованной $(b)$ компонент световой волны 4 от ориентационного угла $\theta$ при $p$-поляризованной волне 2 : точки эксперимент, приведенный в работе [19]; штриховая линия теоретическая кривая, приведенная в [19]; сплошная линия теоретическая кривая, полученная на основании численного решения уравнений связанных волн (3)-(10).

Как видно из построения, периодичности графиков зависимостей $I_{4 p}(\theta)$ и $I_{4 s}(\theta)$, полученных теоретическим и экспериментальным способами, практически совпадают. Вместе с тем при сравнении теоретически рассчитанного графика зависимости с соответствующей экспериментальной кривой можно обнаружить, что в окрестностях локальных максимумов значения интенсивностей $I_{4 p}\left(I_{4 s}\right)$, полученные теоретически и экспериментально, могут существенно отличаться. Например, при использовании $p$-поляризованной волны накачки 2 теоретически рассчитанное значение $I_{4 p}$ в локальном максимуме зависимости $I_{4 p}(\theta)$ (рис. $2, a$ ), достигаемом при $\theta \approx 320^{\circ}$, превышает экспериментально определенное значение $I_{4 p}$ практически в два раза. Аналогичная разность между значениями интенсивности $I_{4 s}$, полученными теоретически и экспериментально при $p$-поляризованной волне накачки 2 , достигается в локальном максимуме графика зависимости $I_{4 s}(\theta)$, соответствующем нулевому значению ориентационного угла (рис. $2, b$ ). При использовании $s$-поляризованной волны накачки 2 наибольшая разница между значениями интенсивностей компонент волны 4, определенными теоретически и экспериментально, заметно меньше. При нахождении интенсивности $s$-поляризованной компоненты волны 4 в случае, когда волна накачки 2 имеет $s$-поляризацию, наибольшее расхождение получено при $\theta \approx 270^{\circ}$ (pис. $3, a$ ): экспериментально найденное значение $I_{4 s}$ больше теоретически рассчитанного приблизительно в 1.45 раза. Наибольшая разность в локальных максимумах графиков $I_{4 p}(\theta)$, полученных теоретически и экспериментально, в случае $s$-поляризованной волны накачки 2 достигается при $\theta \approx 0^{\circ}$. В этом случае теоретически рассчитанное значение $I_{4 p}$ больше экспериментального в 1.7 раза.

Если рассматривать теоретические графики зависимости $I_{4 p}(\theta)$ и $I_{4 s}(\theta)$, полученные в [19], можно видеть, что значения интенсивности в локальных максимумах либо равны друг другу, либо соответствуют двум различным значениям. Например, на рис. $2, a$ значения $I_{4 p}$ в локальных максимумах графика зависимости $I_{4 p}(\theta)$ практически одинаковы и приблизительно равняются $400 \mathrm{nW} / \mathrm{cm}^{2}$. На рис. $2, b$ и рис. 3 значения интенсивностей в локальных максимумах графиков зависимости $I_{4 p}(\theta)$ и $I_{4 s}(\theta)$ равняются $300 \mathrm{nW} / \mathrm{cm}^{2}$ или $120 \mathrm{nW} / \mathrm{cm}^{2}$. Однако это не совсем отвечает приведенным в [19] экспериментальным данным. Как видно из рис. 2 и 3 , значения $I_{4 p}$ и $I_{4 s}$ в локальных максимумах экспериментальных кривых не соответствуют одному или двум фиксированным
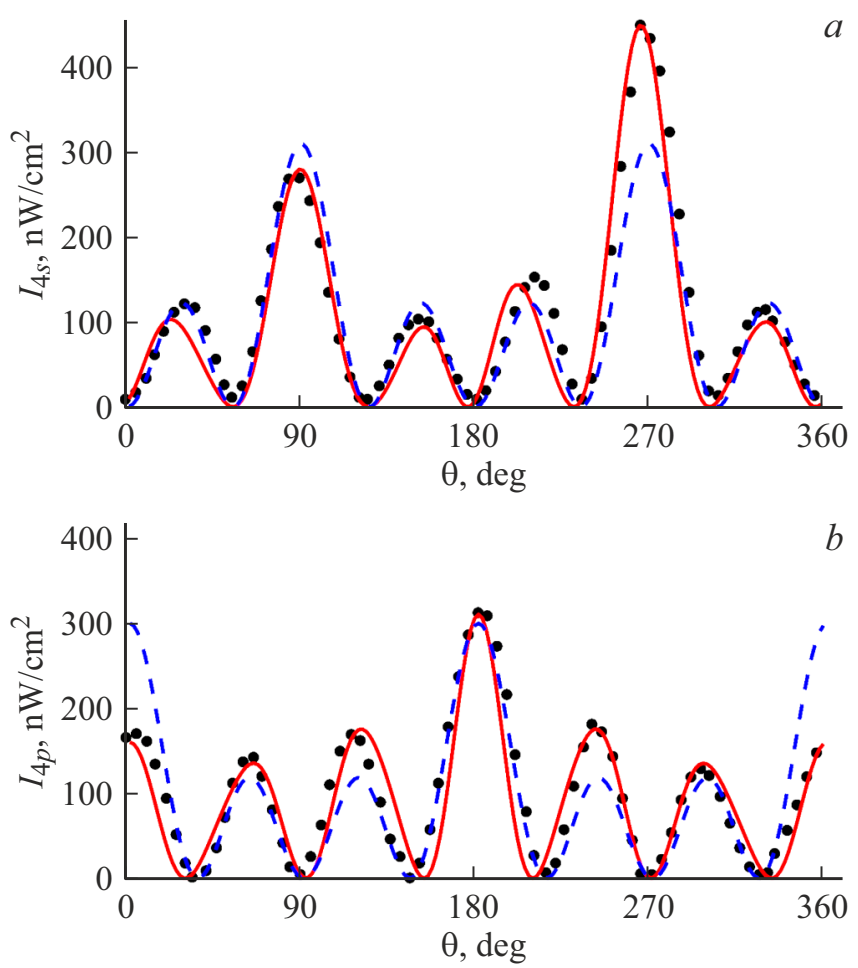

Рис. 3. Зависимости интенсивностей $s$-поляризованной $(a)$ и $p$-поляризованной $(b)$ компонент световой волны 4 от ориентационного угла $\theta$ при $s$-поляризованной волне 2 : точки эксперимент, приведенный в работе [19]; штриховая линия теоретическая кривая, приведенная в [19]; сплошная линия теоретическая кривая, полученная на основании численного решения уравнений связанных волн (3)-(10). 
значениям и могут существенно отличаться друг от друга. Такое различие в теоретических и экспериментальных данных может обусловливаться пренебрежением в теоретической модели рядом свойств кристаллической среды и формируемых в фоторефрактивном полупроводнике голографических решеток. В [19] указывается, что различие в теоретических и экспериментальных данных возможно обусловлено отсутствием учета дифракционного вклада световых волн на амплитудных голографических решетках, формируемых в кристалле GaAs. Вместе с тем необходимо отметить, что в указанной работе не учтена возможность одновременной записи нескольких голографических решеток при встречном четырехволновом взаимодействии в фоторефрактивном кристалле. Кроме того, в уравнениях связанных волн, приведенных в [19], пренебрегалось влиянием фотоупругого и обратного пьезоэлектрического эффектов, которые могут оказывать существенное влияние на поляризационные и энергетические характеристики дифрагированных на голографических решетках световых пучков [27].

На рис. 2 и 3 сплошными линиями отображены графики зависимости $I_{4 p}(\theta)$ и $I_{4 s}(\theta)$, полученные в результате численного решения уравнений связанных волн (3)-(10). При расчете теоретических кривых принимался ряд допущений. Предполагалось, что при четырехволновом взаимодействии формируются 2 пропускающие и 4 отражательные голографические решетки. Допускалось, что пропускающие голографические решетки, которым соответствуют волновые векторы $\mathbf{K}_{13}$ и $\mathbf{K}_{24}$, и отражательные голографические решетки, которым соответствуют волновые векторы $\mathbf{K}_{12}$ и $\mathbf{K}_{34}$, имеют фазово-амплитудную структуру. При взаимодействии волны накачки 2 с сигнальной волной 3 , а также волны накачки 1 с волной 4 происходит формирование отражательных голографических решеток с волновыми векторами, направленными параллельно кристаллографическому направлению [110]. В этом случае фазовые отражательные голографические решетки в кубических фоторефрактивных кристаллах не формируются [28], и при решении уравнений связанных волн (3)-(10) предполагалось, что отражательные решетки являются чисто амплитудными. При расчетах также учитывался совместный вклад фотоупругого и обратного пьезоэлектрического эффектов при формировании голографических решеток, а также поглощение кристалла GaAs.

Как следует из рис. 2 и 3, при численных расчетах совместный учет вышеперечисленных факторов позволяет добиться не только совпадения периодичности теоретических и экспериментальных кривых, но и достигнуть согласования значений интенсивностей поляризационных компонент волны 4 в окрестностях локальных максимумов графиков зависимостей $I_{4 p}(\theta)$ и $I_{4 s}(\theta)$. Например, если при получении уравнений связанных волн (3)-(10) учитывается дифракция световых волн одновременно на нескольких голографических решетках, то это приводит к появлению разброса значений интенсивностей в локальных максимумах графиков зависимостей $I_{4 p}(\theta)$ и $I_{4 s}(\theta)$. За счет соответствующего выбора значений напряженностей электрических полей пространственного заряда в фоторефрактивном полупроводнике и амплитудных постоянных связи можно достигнуть более точного совпадения теоретических и экспериментальных данных. Как показывают результаты численного моделирования, изменение фазовой и амплитудной постоянных связи одной из шести голографических решеток не обязательно приводит к изменению интенсивности одновременно во всех локальных максимумах графика зависимости. Как правило, варьирование фазовой и амплитудной постоянных связи голографической решетки приводит к изменению значений $I_{4 p}$ и $I_{4 s}$ в локальных максимумах, симметрично расположенных относительно значений ориентационных углов.

Учет в уравнениях связанных волн совместного вклада фотоупругого и обратного пьезоэлектрического эффектов оказывает существенное влияние на графики зависимости $I_{4 p}(\theta), I_{4 s}(\theta)$ и является одним из условий успешного согласования теоретических и экспериментальных кривых. Под действием этих эффектов в зависимости от ориентационного угла может иметь место как увеличение величины интенсивности поляризационной компоненты волны 4, так и ее уменьшение. При этом происходит небольшое (порядка нескольких градусов) изменение значений ориентационного угла, при которых достигаются локальные максимумы графиков зависимостей $I_{4 p}(\theta)$ и $I_{4 s}(\theta)$. При „включении“ в уравнениях связанных волн (3)-(10) поглощения кристалла имеет место количественное уменьшение интенсивности поляризационной компоненты волны 4. Наибольшее уменьшение интенсивности достигается в окрестностях локальных максимумов графиков зависимостей $I_{4 p}(\theta)$ и $I_{4 s}(\theta)$. В окрестностях локальных минимумов интенсивность практически не изменяется. Качественной трансформации графиков зависимостей $I_{4 p}(\theta)$ и $I_{4 s}(\theta)$ при „включении“ поглощения кристалла не отмечено.

\section{Заключение}

Представлена система уравнений связанных волн, пригодная для расчета вырожденного встречного четырехволнового взаимодействия на 6 голографических (2 пропускающих и 4 отражательных) фазово-амплитудных голографических решетках, сформированных в кубическом фоторефрактивном кристалле класса симметрии $\overline{4} 3 m$. При составлении математической модели рассматривался случай, когда падающие на кристалл световые волны имеют линейную поляризацию, а кристаллическая решетка имеет произвольную ориентацию относительно голографического стола. В математической модели принимался во внимание совместный вклад 
линейного электрооптического, фотоупругого и обратного пьезоэлектрического эффектов, а также учтено поглощение кристалла.

На основании сравнения результатов численного решения полученных уравнений связанных волн с известными экспериментальными данными показано, что более точные результаты расчета интенсивности поляризационных компонент обращенной волны достигаются при аккуратном учете вкладов всех дифракционных процессов, происходящих на пропускающих и отражательных голографических решетках, которые формируются при встречном четырехволновом взаимодействии в фоторефрактивном кристалле. При этом необходимо принимать во внимание, что в фоторефрактивном полупроводнике могут формироваться голографические решетки с фазово-амплитудной структурой. Учет фотоупругого эффекта совместно с обратным пьезоэлектрическим эффектом и поглощением кристалла приводит к более точному предсказанию локальных максимумов графиков зависимостей интенсивности поляризационных компонент обращенной волны от ориентационного угла.

\section{Финансирование работы}

Работа выполнена при финансовой поддержке Министерства образования Республики Беларусь (договор от 22.03.2021 № 1410/2021) в рамках Государственной программы научных исследований № 6 ,Фотоника и электроника для инноваций“ на 2021-2025 гг. (задание 6.1.14).

\section{Список литературы}

[1] В.М. Петров, А.В. Шамрай. Интерференция и дибракиия для интерференционной фотоники (Лань, СПб., 2019).

[2] С.Г. Одулов, М.С. Соскин, А.И. Хижняк. Лазеры на динамических решетках: оптические генераторы на четырехволновом смешении (Наука, М., 1990).

[3] М.П. Петров, С.И. Степанов, А.В. Хоменко. Фоторефбрактивные кристаллы в когерентной оптике (Наука, СПб., 1992).

[4] M.B. Klein. Opt. Lett., 9 (8), 350 (1984).

[5] A.M. Glass, A.M. Johnson, D.H. Olson, W. Simpson, A.A. Ballman. Appl. Phys. Lett., 44 (10), 948 (1984).

[6] M.B. Klein, S.W. Mc Cahon, T.F. Boggess, G.C. Valley. J. Opt. Soc. Am. B., 5 (12), 2467 (1988).

[7] H. Rajbenbach, B. Imbert, J.P. Huignard, S. Mallick. Opt. Lett., 14 (1), 78 (1989).

[8] G. Gheen, L.-J. Cheng. Appl. Phys. Lett., 51 (19), 1481 (1987).

[9] R. Nietzke, P.Panknin, W. Elsässer, E.O. Göbel. IEEE J. Quant. Electron., 25 (6), 1399 (1989).

[10] T. Sadeev, H. Huang, D. Arsenijevic, K. Schires, F. Grillot, D. Bimberg. Appl. Phys. Lett., 107, 191111 (2015).

[11] I.A. Solovev, Yu.V. Kapitonov, B.V. Stroganov, Yu.P. Efimov, S.A. Eliseev, S.V. Poltavtsev. J. Physics: Conf. Series, 1124, 051042 (2018)
[12] K. Je, K. Kyhm. Rapid Res. Lett., 1800354 (2018).

[13] А.А. Изванов, А.Е. Мандель, Н.Д. Хатьков, С.М. Шандаров. Автометрия, 2, 79 (1986).

[14] С.И. Степанов, С.М. Шандаров, Н.Д. Хатьков. ФТТ., 29 (10), 3054 (1987).

[15] H. Kogelnik. Bell Syst. Tech. J., 48, 2909 (1969).

[16] G. Montemezzani, M. Zgonik. Phys. Rev. E., 55(1), 1035 (1997).

[17] В.В. Шепелевич, А.В. Макаревич, С.М. Шандаров. Письма в ЖТФ, $40(22), 83$ (2014). [V.V. Shepelevich, A.V. Makarevich, S.M. Shandarov. Tech. Phys. Lett., 40 (11), 1024 (2014)].

[18] R.B. Bylsma, D.H. Olson, A.M. Glass. Opt. Lett., 13 (10), 853 (1988).

[19] Y. Ding, H.J. Eichler. Opt. Comm., 110, 456 (1994).

[20] Р.В. Литвинов, С.И. Полковников, С.М. Шандаров. Квант. электрон., 31 (2), 167 (2001). [R.V. Litvinov, S.I. Polkovnikov, S.M. Shandarov. Quant. Electron., 31 (2), 167 (2001)].

[21] А.В. Гусельникова, С.М. Шандаров, А.М. Плесовских, Р.В. Ромашко, Ю.Н. Кульчин. Опт. журн., 73 (11), 22 (2006). [A.V. Gusel'nikova, S.M. Shandarov, A.M. Plesovskikh, R.V. Romashko, Yu.N. Kulchin. J. Opt. Technol., 73 (11), 760 (2006)].

[22] В.Н. Навныко, С.Ф. Ничипорко, А.В. Макаревич, C.M. Шандаров. ЖТФ., 91 (5), 832. (2021). [V.N. Naunyka, S.F. Nichiporko, A.V. Makarevich, S.M. Shandarov. Tech. Phys., 66 (5), 808 (2021)].

[23] С.М. Шандаров, В.В. Шепелевич, Н.Д. Хатьков. Опт. и спектр., $\mathbf{7 0}(5), 1068$ (1991). [S.M. Shandarov, V.V. Shepelevich, N.D. Khatkov. Opt. Spectrosc., 70 (5), 627 (1991)].

[24] K. Shcherbin, S. Odoulov, R. Litvinov, E. Shandarov, S. Shandarov. J. Opt. Soc. Am. B., 13 (10), 2268 (1996).

[25] A. Dargys, J. Kundrotas. Handbook on physical properties of Ge, Si, GaAs and InP (Science and Encyclopedia Publishers, 1994).

[26] Y.H. Ja. Opt. and Quant. Electron., 15, 539 (1983).

[27] V.V. Shepelevich, N.N. Egorov, V. Shepelevich. J. Opt. Soc. Am. B., 11 (8), 1394 (1994).

[28] V.V. Shepelevich, V.N. Naunyka. J. Holography Speckle., 5 (3), 286 (2009). 